\title{
Carrier relaxation dynamics in heavy fermion compounds
}

\author{
J. Demsar*, L.A. Tracy, R.D. Averitt, S.A.Trugman, J.L.Sarrao, A.J. Taylor \\ Los Alamos National Laboratory, Mail Stop K764, Los Alamos, NM 87545, USA
}

\begin{abstract}
The first femtosecond carrier relaxation dynamics studies in heavy fermion compounds are presented. The carrier relaxation time shows a dramatic hundred-fold increase below the Kondo temperature revealing a dramatic sensitivity to the electronic density of states near the Fermi level.
\end{abstract}

\section{INTRODUCTION}

Femtosecond time-resolved optical spectroscopy is an excellent experimental alternative to conventional spectroscopic methods that probe the low energy electronic structure in strongly correlated electron systems [1]. In particular, it has been shown that carrier relaxation dynamics are very sensitive to changes in the low energy density of states (e.g. associated with the formation of a low energy gap or pseudogap) providing new insights into the low energy electronic structure in these materials. In this report we present the first studies of carrier relaxation dynamics in heavy fermion (HF) systems by means of femtosecond time-resolved optical spectroscopy. Our results show that the carrier relaxation dynamics, below the Kondo temperature $\left(\mathrm{T}_{K}\right)$, are extremely sensitive to the low energy density of states (DOS) near the Fermi level to which localized f-moments contribute. Specifically, we have performed measurements of the photoinduced reflectivity $\Delta R / R$ dynamics as a function of temperature and excitation intensity on the series of $\mathrm{HF}$ compounds $\mathrm{YbXCu}_{4}(\mathrm{X}=\mathrm{Ag}, \mathrm{Cd}, \mathrm{In})[2]$ in comparison to their non-magnetic counterparts $\mathrm{LuXCu}_{4}$.

\section{EXPERIMENTAL}

The experiments were performed on freshly polished flux-grown single crystals. We used a standard pump-probe set-up with a mode-locked Ti:Sapphire laser (30 fs pulses at $800 \mathrm{~nm}$ and $80 \mathrm{MHz}$ repetition rate) for both pump and probe pulse trains. The photoinduced (PI) change in reflectivity $\Delta \mathrm{R} / \mathrm{R}$ was measured using photodiode and lock-in detection. The pump laser fluence was kept below $10 \mu \mathrm{J} / \mathrm{cm} 2$ and the pump/probe intensity ratio was $\sim 30$. The steady-state heating effects were accounted for as described in [3] yielding an uncertainty in temperature of $\pm 3 \mathrm{~K}$.

\section{RESULTS AND DISCUSSION}

In Fig. 1a we show the PI reflectivity traces as a function of temperature on the HF system $\mathrm{YbCdCu}_{4}\left(\mathrm{~T}_{K}\right.$ $\sim 100 \mathrm{~K}$, low temperature Sommerfeld coefficient $\gamma \sim 200 \mathrm{~mJ} / \mathrm{mol} \mathrm{K}^{2}$ ). The relaxation time is virtually temperature independent above $\sim 140 \mathrm{~K}$ and shows a quasi-divergence (hundred-fold increase) as $\mathrm{T}=0 \mathrm{~K}$ is approached.

Similar behavior (see Fig. 1b) is observed also in $\mathrm{YbAgCu}_{4}\left(\mathrm{~T}_{K} \sim 100 \mathrm{~K}, \gamma \sim 200 \mathrm{~mJ} / \mathrm{mol} \mathrm{K}^{2}\right.$ ) [2]. Importantly, $\mathrm{YbAgCu}_{4}$ is a much better metal than $\mathrm{YbCdCu} 4$ revealing that the observed dynamics are not merely due to changes in the carrier concentration. Furthermore, the relaxation time of the non-HF compound $\mathrm{LuAgCu}_{4}$ behaves similar to regular metals 何, showing a gradual decrease upon cooling. This, coupled with the data on the HF compounds in Fig. $1 \mathrm{~b}$, indicates that the upturn in the relaxation time at $\sim \mathrm{T}_{K}$ and the subsequent divergence as $\mathrm{T} \rightarrow 0 \mathrm{~K}$ derives from the low energy electronic structure of these HF compounds.

An important and unresolved issue in HF physics $[5]$ is whether the electronic ground state is a many-body Kondo state that forms only below $\mathrm{T}_{K}$, or is more simply described in terms of the thermal population of a narrow gap system with the gap arising from hybridization between the conduction electrons and localized $f$ electrons (note that the gap is present at all temperatures above and below $\mathrm{T}_{K}$ in this case). As we have shown, the ultrafast dynamics are clearly sensitive to the low energy electronic state and such experiments may provide insight into the true nature of the HF ground state. However, a complete analysis (currently in progress) requires many-body calculations beyond the scope of this paper. In the following, we present a heuristic model, which provides some physical insight into the low temperature electron dynamics in HF compounds.

The two-temperature model is commonly employed to interpret the ultrafast dynamics in metals 四 with the sequence of relaxation events after photoexcitation as follows: the photoexcited carriers first rapidly thermalize via electron-electron scattering $\left(\tau_{e-e} \approx 100 \mathrm{fs}\right)$, giving rise to a rapid change in reflectivity on a sub-picosecond timescale. This is followed by energy transfer to the lattice with a characteristic electron-phonon relaxation time $\tau_{e-p h}$ on the order of a picosecond [4]. At low excitation fluences, as used here, the increase in the electronic temperature after photoexcitation is small compared to the lattice temperature, T. In this limit the T-dependence of $\tau_{e-p h}$ in the 
FIG. 1: a) Normalized photoinduced reflectivity in $\mathrm{YbCdCu}_{4}$ at various temperatures above and below $\mathrm{T}_{K} \sim 100 \mathrm{~K}$. b) Temperature dependence of the relaxation time $\tau_{R}$ for two different HF compounds, together with the power-law fits $\tau_{R}=T^{-p}$, where $\mathrm{p}=3 \pm 0.5$. Note that the dotted line for $\mathrm{LuAgCu} 4$, which is not a HF compound, does not display this power law behavior. c) Temperature dependence of the Sommerfeld constant $\gamma$ for $\mathrm{YbAgCu}_{4}$ as extracted from the relaxation time data (solid squares) compared to the calculated $\gamma$ for the case of a single impurity model [6] with the impurity spin $7 / 2$.

two-temperature model is given by $\tau_{e-p h}=\gamma(T) T / g(T)$, where $g(T)$ is the electron-phonon coupling function [ $[$. Since $g(T)$ is constant above the Debye temperature $\Theta_{D}$, and proportional to $\mathrm{T}^{4}$ at $\mathrm{T}<<\Theta_{D}, \tau_{e-p h}$ is expected to decrease linearly upon cooling from room temperature, showing a minimum with a subsequent increase going as $\mathrm{T}^{-3}$ at low temperatures. However, this low- $\mathrm{T}$ upturn in $\tau_{R}$ is not observed in $\mathrm{LuAgCu}_{4}$ (Fig $1 \mathrm{~b}$ ) - as it was not observed in conventional Fermi liquid metals like $\mathrm{Au}$ and $\mathrm{Ag}$ 㽢. The lack of the low- $\mathrm{T}$ quasi-divergence in $\tau_{R}$ in conventional metals was shown to result due to the fact that $\tau_{e-e}$ and $\tau_{e-p h}$ are comparable below $\approx 300-600 \mathrm{~K}$ [4] in the experimental configuration of low laser powers. A non-thermal electron model [i] that takes into account a "finite" $\tau_{e-e}$ can account for a) the increased room temperature $\tau_{e-p h}$ and b) the low temperature plateau in the observed temperature dependence of $\tau_{e-p h}$ in $\mathrm{Au}$ and $\mathrm{Ag}$ 国 as well as in $\mathrm{LuAgCu}_{4}$.

However, in the HF compounds we have studied $\gamma$ is, below $\mathrm{T}_{K}$, two orders of magnitude larger than in conventional metals. This is expected to give rise to a hundred-fold increase in $\tau_{e-p h}$ in these systems and the two-temperature model (without considering the initial non-thermal electron distribution) is expected to be valid. This would give rise to $\tau_{R} \propto T^{-3}$ at low-T as experimentally observed (Fig. 1b). Furthermore, since $g(T)$ depends only on $\Theta_{D}$ and a prefactor that can be estimated from the high-T value of $\tau_{R}$ 泊 (at $300 \mathrm{~K}$ the value of $\tau_{R}$ is close to the estimated $\tau_{e-p h}$ ), one can extract the Sommerfeld constant via $\gamma(T)=g(T) \tau_{R} / T$. We find a remarkable agreement between the low temperature $\gamma(T)$ extracted in this way from $\tau_{R}(T)$ and the theoretical calculation [6] for the case of single impurity model with impurity spin $7 / 2$ - Fig 1c, giving further support to this analysis.

\section{CONCLUSIONS}

In conclusion, we have utilized ultrafast optical techniques to study the dynamics of quasiparticles in HF compounds $\mathrm{YbXCu}_{4}$. We have observed a power-law divergence in the relaxation time at low temperatures. The dramatic hundred-fold increase in the relaxation time at low temperatures in $\mathrm{YbXCu}_{4}$ (and the lack of this quasi-divergence in the non-HF $\mathrm{LuXCu}_{4}$ analogs) clearly demonstrates that our measurements probe the heavy fermion ground state. 
* phone: 1-505-665-8839, fax 1-505-665-7652, e-mail: jdemsar@lanl.gov

[1] V.V.Kabanov et al., Phys.Rev.B 59, (1999) 1497, J.Demsar, D.Mihailovic, K.Biljakovic, Phys.Rev.Lett. 83,800 (1999).

[2] T.Graf et al., Phys. Rev. B 51 (1995) 15053; J. L. Sarrao et al., Phys. Rev. B 59 (1999) 6855.

[3] D. Mihailovic and J. Demsar in Spectrosopy of Superconducting Materials, ed. Eric Falques, ACS Symposium Series 730; The American Chemical Society: Washington, D.C., 1999.

[4] R. H. M. Groeneveld, R. Sprik, A. Lagendijk, Phys. Rev. B 51 (1995) 11433, and the references therein.

[5] L. Degiorgi, Rev. Mod. Phys. 71, 687 (1999) and the references therein.

[6] V.T.Rajan, Phys.Rev.Lett.51 (1983) 308. 


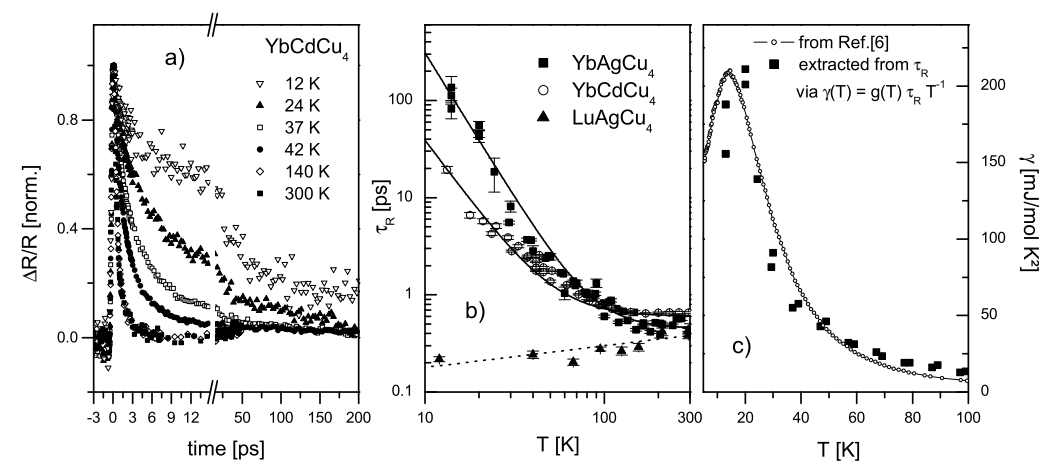

